DEPARTMENT OF HEALTH (1996) Mental Health Act 1983 Code of Practice. Revision. London: Department of Health.

opinion \& debate
GENERAL MEDICAL COUNCIL (1999) Seeking Patients' Consent: The Ethical Considerations. London: GMC

IOHNSON, C, SMITH, J. STAINER, G. (1993) Mildly mentally handicapped offenders: an alternative to custody. Psychiatric Bulletin, 17, 199-201.
LARKIN, M. (1997) Approaches to amelioration of autism in adulthood. Lancet, 349, 186

SMITH, J. (1988) An open forensic unit for the borderline mentally impaired offender. Psychiatric Bulletin, 12, 13-15. WORLD PSYCHIATRIC ASSOCIATION (1981) The Declaration of Hawaii. Adopted by the General Assembly in Honolulu 1977. Psychiatric Ethics (eds S. Bloch \& P. Chedoff), pp. 524-526.

Oxford: Oxford University Press.
Lord Donaldson MR in ReJ (a minor) (medical treatment) (1992) 4 all ER 614.

Re C Adult Refusal of Medical treatment (1994) 1 All ER 819

L v. Bournewood Community and Mental Health NHS Trust decision by the House of Lords in the appeal. Department of Health (1998).

John J. Sandford Specialist Registrar in Forensic Psychiatry, Leader Unit, Langdon Hospital, Exeter Road, Dawlish, Devon EX7 0NR

Ernest Gralton Consultant in Forensic Learning Disability Psychiatry,

St Andrew's Hospital, Northampton, W. M. Donovan Consultant Forensic

Psychiatrist, Langdon Hospital, Dawlish

\title{
HARRY KENNEDY
}

\section{Risk assessment is inseparable from risk management}

\author{
Comment on Szmuckler (Psychiatric Bulletin, 2000, 24, 6-10)
}

Szmuckler (2000) has argued that patients in the community represent such low risks of grave outcomes that it is impossible to predict harm accurately. Others have made the same case for the impossibility of predicting suicide (Geddes, 1999). Based on a 12-month prevalence of psychosis of 4 in 1000 (or 200000 cases in England and Wales) and a homicide rate by people with schizophrenia (or severe mental illness) of approximately 20 per year in England and Wales (Taylor \& Gunn, 1999), Szmuckler arrives at a homicide rate of 1 per 10000 'psychosis years'. This is about 10 times the average annual homicide rate for all of England and Wales (Kennedy et al, 1999). At such low rates Szmuckler calculates that even predictions with sensitivity of $90 \%$ (the proportion of true negative predictions) would give 2000 false positives for every homicide accurately predicted. A recalculation employing Szmuckler's assumptions, using a standard probability matrix (Bourke et al, 1985, p 248) gives 1111 false positives per homicide accurately predicted (Table 1)

\section{Violence, not homicide}

Recorded crimes of violence are much more common than homicide, by a factor of about 200 for the general population of London (Kennedy et al, 1999) and the real rate of violence in the community is even greater. Anyone familiar with witness statements will know that the difference between an assault and a homicide is often merely a matter of chance. Violence, because it is more common, should be easier to predict than homicide. There are about 1000 hospital orders, restricted and unrestricted (Sections 37 and 37/41) per year in England and Wales, with a further 250 transfers from prison under Section $47 / 49$ (Kershaw \& Renshaw, 1998). These are not all sentenced for crimes of violence, but most are, and a further number of patients with severe mental illness are dealt with by making civil orders in Magistrates' Courts (James, 1999) or by the police bringing patients directly to hospital under Section 138 (Humphries \& Johnstone, 1993). An annual estimate of 4000 recorded crimes of violence by people with mental illness seems reasonable in this context.

Using the same assumed 12 -month prevalence of schizophrenia, at $90 \%$ sensitivity and $90 \%$ specificity, Table 1 shows that there would be 4.95 false predictions of reported crimes of violence per accurate prediction. This would lead to the targeting of extra services to $11.8 \%$ of patients. Violence should therefore be easier to predict than homicide or suicide, but even so, Szmukler's use of homicide as an outcome measure is a straw-man argument with several related, but mistaken, assumptions.

Table 1. Homicide rate in 'psychosis years' using a standard probability matrix

\begin{tabular}{|lccc}
\hline & $\begin{array}{c}\text { Homicide (violence) } \\
\text { actually occurs }\end{array}$ & $\begin{array}{c}\text { Homicide (violence) } \\
\text { does not occur }\end{array}$ & $\begin{array}{c}\text { Row totals } \\
\text { Homicide matrix (violence matrix) }\end{array}$ \\
\hline Homicide predicted (violence predicted) & $(\mathrm{a})$ & (b) & \\
& $18(3600)$ & $19998(19960)$ & $(23560)$ \\
Homicide not predicted (violence not predicted) & $2(400)$ & $179982(176040)$ & $179984(176440)$ \\
$\begin{array}{l}\text { Column totals } \\
\text { Homicide matrix (violence matrix) }\end{array}$ & $20(4000)$ & $199980(196000)$ & $200000(200000)$ \\
\hline
\end{tabular}

Based on Szmuckler's assumptions (Szmuckler, 2000). Note: sensitivity (ability to detect true cases) $=a /(a+c)=90 \%$. Specificity (ability to detect true non-cases) $=d /(b+d)=90 \%$. Homicide false positives per correct prediction $=19998 / 18=1111$. Violence false positives per correct prediction=19 960/3600=5.54. 
Homicide (violence) Homicide (violence) risk present

Preventive measures applied

Homicide (violence)

Preventive measures not applied

Homicide (violence)

Column totals

Homicide matrix (violence matrix)

$20(4000)$

$179802(15600)$

$220(44000)$

$199780(156000)$ risk not present

Row totals

Homicide matrix (violence matrix)

$\begin{array}{ccc}200(40000) & 19978(15600) & 20178(55600) \\ \text { (c) } & \text { (d) }\end{array}$

$179822(144400)$

$200000(200000)$

Alternative assumptions: (a) those correctly assessed as at risk who receive effective preventive measures; (b) those incorrectly assessed as at risk who also receive preventive measures; (c) those incorrectly assessed as not at risk, or not known to services, who do not receive preventive measures, and (d) those correctly assessed as not at risk who also do not receive preventive measures.

Inappropriate application of preventive measures per homicide prevented $=19$ 978/200=99.9. Per act of violence prevented $=15600 / 40000=0.39$

\section{Homicide and violence as system failures}

Szmuckler has assumed that no risk assessment or risk management is currently in place. It would be more accurate to assume, at least for the sake of probability modelling, that the 20 homicides a year by those suffering from severe mental illness, like the 4000 violent offences, represent the failure rate of a mental health system for combined risk assessment and risk management, considered at the population level. Table 2 sets out sensitivity and specificity matrices for risk management in which patients either have or have not a risk of violence and homicide that for the sake of simplicity is either 0 (no risk at all) or 1 (certainty) for the coming year. Similarly, the process of risk management (a combination of clinical risk assessment, risk reduction through treatment and long-term risk management through the therapeutic uses of security) is assumed, for the sake of simplicity, to be $100 \%$ effective when applied. If the overall system, considered at the population level, has sensitivity and specificity of $90 \%$ such that 20 homicides and 4000 recorded crimes of violence would follow, then 100 patients are inappropriately treated for each homicide prevented. But only two are inappropriately treated for every five crimes of violence prevented.
Since the therapeutic use of security includes a wide range of short- medium- and long-term interventions, including not only detention in secure hospitals, but also 24-hour nursed care units, high support hostels and assertive community care teams, the treatments inappropriately applied are not necessarily onerous and will have other health benefits.

\section{Risk assessment and stratified therapeutic security as a system for risk management}

Of course in practice, the risk of violence is a continuous variable rather than all or nothing, and no system of mental health care could be $100 \%$ effective in preventing violence by those with severe mental illness, in the community or in hospital. Table 3 shows a risk matrix that assumes that two groups of patients can be distinguished, one with a mean risk of violence in the next year of 0.5 (high) and the other with a mean risk of 0.02 (the population risk in community surveys). It is also assumed that clinical preventive measures, broadly defined, are $99 \%$ effective. In this model, the same number of recorded crimes of violence will follow if the mental health system operates with a sensitivity and specificity

Table 3. Risk matrix assuming two groups of patients (high and low risk) can be distinguished

\begin{tabular}{|c|c|c|c|}
\hline & $\begin{array}{l}\text { Violence high risk } \\
\text { present }(P=0.5)\end{array}$ & $\begin{array}{l}\text { Violence low risk present } \\
\qquad(P=0.02)\end{array}$ & Row totals \\
\hline Preventive measures applied (0.99 effective) & $\begin{array}{l}9542 \text { (48 episodes } \\
\text { of violence occur) } \\
\text { (c) }\end{array}$ & $\begin{array}{l}19130 \text { (4 episodes } \\
\text { of violence occur) } \\
\text { (d) }\end{array}$ & 28672 \\
\hline \multicolumn{4}{|l|}{ Column totals } \\
\hline & $\begin{array}{c}10626 \text { (590 episodes } \\
\text { of violence occur) }\end{array}$ & $\begin{array}{c}189374 \text { (3409 episodes } \\
\text { of violence occur) }\end{array}$ & $\begin{array}{c}200000 \text { (3999 episodes } \\
\text { of violence occur) }\end{array}$ \\
\hline \multicolumn{4}{|c|}{$\begin{array}{l}\text { Assuming those at high risk have an average probability of violence of } 0.5 \text {, while those at low risk have an average probability of } 0.02 \text {; preventive measures are assumed to } \\
\text { have a failure rate of } 0.01 \text {. Sensitivity }=90 \% \text {; specificity }=90 \% \text {. } 3999 \text { episodes of violence would occur ( }(\mathrm{a}) \times 0.5 \times 0.01 \text { plus (b) } \times 0.02 \times 0.01 \text { etc., and } 5101 \text { episodes of } \\
\text { violence would be prevented (a) } \times 0.5 \times 0.99 \text { plus (b) } \times 0.02 \times 0.99 \text {. Twenty-eight thousand, six hundred and seventy-two patients would receive preventive measures of } \\
\text { whom } 23570 \text { would be inappropriately treated ( } 4.6 \text { inappropriately treated for each episode of violence prevented, or the number needed to treat to prevent one episode } \\
\text { of violence }=5.6 \text { ). }\end{array}$} \\
\hline
\end{tabular}


of $90 \%$, while 5.6 patients receive preventive measures (4.6 inappropriately) for each episode of violence prevented.

opinion

\& debate

\section{Theoretical and practical considerations}

The model illustrated in Table 3 goes some way towards compensating for the deficiencies in the model employed by Szmuckler. The assumption that patients are not assessed for risk, and that their clinical management is not modified to take account of this, is common in existing research studies of risk assessment, for example, Powell et al (2000) and in many of the studies reviewed by Dolan and Doyle (2000).

\section{Placement decisions are made to stratify patients according to risk}

It is wrong to assume that all groups of patients really are at the average risk for patients nationally. Even in nonforensic populations presenting unselected from the community, it is a commonplace observation that about $3 \%$ of the patients account for about $50 \%$ of the violence in hospital (Crichton, 1996). All mental health services, general as well as forensic, respond by carefully stratifying patients according to the risk they present to themselves and to others. Snowden et al (1999) point out that three-quarters of all restriction order patients in England and Wales (2744 and 3677, respectively) are in hospital at any one time. Even for populations of patients defined by local sectors, the most high risk will be in hospital owing to acute relapses, and for inner-city populations a higher proportion will be in secure forensic units (Glover et al, 1999). Within forensic services, the aim is to stratify patients according to the risk of violence and the gravity of the violence. This allows the therapeutic use of security (relational, procedural and environmental) to be matched (high, medium, low, etc.) to the current need, determining the pace of progress through treatment settings, from admission to rehabilitation to community placement. Forensic patients are selected for discharge when stable, predictable and willing to tolerate a degree of intrusion and control by clinicians in the community. In community forensic populations the average risk of grave violence is higher than for patients in general psychiatric community services.

\section{Risk is stratified in time}

The risk of suicide is greatest in the year after discharge from hospital, of the order of 48 to 61 per 10000 patient years (Fawcett et al, 1990; Geddes et al 1997; Rossau \& Mortensen, 1997). In a recent US study, the risk of violence in the year following hospital discharge was 1990/10 000 (19.9\%) for patients with mental illness without substance misuse problems, and 3110/10 000 for those with mental illness and comorbid substance misuse (Steadman et al, 1998). This temporal stratification of risk should enable more accurate risk assessments for the period immediately after leaving hospital because of the higher average risk during that period. More intensive contact with mental health services (relational and procedural security) might be expected to manage the risk within the bounds of what is possible or predictable.

\section{The variance of risk}

The risk of violence or suicide is a statistically distributed variable in any population, whether or not there are distinct sub-populations with distinct distributions owing to disease or other factors. A wide variance in the distribution of risk can weaken the sensitivity and specificity of any method of assessment. However, just as it is wrong to assume that all patient populations are at the national average for a given risk, it is wrong to assume that the average risk in any patient group is made up of the same wide range of risk. Patients new to a mental health service are at unknown average risk and probably have a wide range of risk. In forensic and other services, patients are selected to stratify according to level of risk and gravity of the risk, but this stratification will also narrow the range of risk within each group. Those treated in the community should also be selected within a narrow range of risk. This narrow range should increase the accuracy of risk assessment.

\section{Contextual factors}

Clinical judgements and predictions are not made in a statistically pure vacuum. Such judgements are contextual, they are revised in the light of relevant information and they are often very specific to the experience of the clinician and of the patient. Bayesian approaches to measurement of risk are a better model for such assessments, in which probability is regarded as representing a degree of reasonable belief (Hey, 1983). Powell et al (2000) employ Bayesian corrections for the likelihood ratios of risk-factors identified in their study so that the local context is included.

An important caveat regarding risk prediction concerns the assumption that all local populations from which patients are drawn are at the same average risk as the total population. This is untrue. In inner-city boroughs the population risk of homicide, suicide or recorded crimes of violence increases exponentially with population density or deprivation (Kennedy et al, 1999). This variable background of environmental risk probably worsens the risk of adverse outcomes such as violence, homicide or suicide for a given patient. The non-linear relationship of the outcome to its (assumed) environmental determinants probably limits the clinician's capacity to make anything more than short-term predictions when the environmental risk is high.

\section{Systems failure and the therapeutic uses of security}

From admission ward through to locked intensive care to rehabilitation ward, 24-hour nursed care and hostel, all constituent parts of a mental health hospital service systematically stratify patients according to a hierarchy of 
risk, so that patients are allocated to places with high, medium or low levels of relational, procedural and environmental security. This long-standing structural risk management at the systems level may have been lost in modern sectorised community mental health teams and small generic in-patient units. If general psychiatrists' daily manage mixed populations of patients, many of whom are at a risk of committing homicide of 1/10 000 per year as Szmuckler suggests, (or 200/10 000 risk of violence) they may be unaware that a few of their patients are at a risk of 1/100 for homicide or 1/10 for violence. Even with little regard for risk management, most psychiatrists responsible for catchment populations of 30000 to 50000 (120 to 200 with severe mental illness) would still seldom experience adverse outcomes and may be unaware of the risks they run in some cases. This functional risk-blindness is likely to be particularly hazardous in high risk inner-city boroughs. It follows that the mixing of all patients from a small sector catchment area in small generic services is bad risk management at the systems level. Not only does it prevent the targeting of specialised care by stratifying according to need, it can make risk assessment less accurate and risk management too diffused.

\section{Conclusions}

The figures given by Szmuckler, and in Tables 1-3, depend on estimates and inferences. It would be possible to give real figures for the risk management matrices based on case registers and hospital activity data if health service information was accurately collated (Glover, 2000). The criminal justice data could similarly be collated. The use of presentation rates via the criminal justice system as a performance measure for mental health services is already possible (Purchase et al, 1996).

The prevailing rate of homicide in the community by patients with schizophrenia is already modified by risk stratification in mental health services. Szmuckler is right to observe that there is little point in focusing on the prevention of homicide. But this is because the relationship between acts of violence and completed homicide is often a matter of bad luck. Risk assessment is worth the effort and can be improved on, although only by recognising that the process of assessment is, for practical purposes, inseparable from the process of managing risk by matching the patient's risk to the appropriate level of therapeutic security. The risk of violence should be more predictable and more easily managed, within the limits of what is possible with the available resources and the risks inherent in the local community. What really matters is the effectiveness of the mental health services as a risk management process for the patient population overall.

\section{References}

BOURKE, G. J., DALY, I. E. \& KENNEDY, H. G., IVESON, R. Y. \& HILL, O MCGILVRAY, J. (1985) Interpretation and Uses of Medical Statistics (3rd edn) Oxford: Blackwell Scientific.

CRICHTON, J. (1996) Psychiatric inpatient violence, Appendix A. In Dangerous People (ed. N. Walker), pp.144-145. London: Blackstone Press.

DOLAN, M. \& DOYLE, M. (2000) Violence risk prediction. Clinical and actuarial measures and the role of the Psychopathy Checklist. British Journal of Psychiatry, 177, 303-311.

FAWCETT, J., SCHEFTNER, W. A., FOGG, L., et al (1990) Time-related predictors of suicide in major affective disorder. American Journal of Psychiatry, 147, 1189-1194

GEDDES, J. (1999) Suicide and homicide by people with mental illness. British Medical Journal, 318, 1125-1226.

- JUSZACK, E., O'BRIEN, F., et al (1997) Suicide in the 12 months after discharge from psychiatric in-patien care, Scotland 1968-92. Journal of Epidemiology and Community Health, 51, 430-434.

GLOVER, G. (2000) The minimum data set. At last - information! Psychiatric Bulletin, 24, 163-164.

—, LEESE, M. \& MCCRONE, P. (1999)

More severe mentalillness is more concentrated in deprived areas. British Journal of Psychiatry, 175, 544-548.

HEY, J. (1983) Data in Doubt. Oxford: Martin Robertson.

HUMPHRIES, M. \& JOHNSTONE, E. C. (1993) Dangerous behaviour preceding first admissions for schizophrenia. British Journal of Psychiatry, 163, 547

JAMES, D. (1999) Court diversion at ten years: can it work, does it work and has it a future? Journal of Forensic

Psychiatry, 10, 507-524.

Harry Kennedy Consultant Forensic Psychiatrist, Central Mental Hospital,

Dundrum, Dublin 14, Ireland; e-mail: harry.kennedy@ireland.com strong correlation and wide variation across districts. British Journal of Psychiatry, 175, 462-466.

KERSHAW, C. \& RENSHAW, G. (1998) Statistics of mentally disordered offenders, England \& Wales 1997 Home Office Statistical Bulletin, 19

POWELL, J., GEDDES, J., DEEKS, J., et al (2000) Suicide in psychiatric hospital inpatients. Risk factors and their predictive power. British Journal of Psychiatry, 176, 266-272.

PURCHASE, N. D., MCCALLUM, A. K. \& KENNEDY, H. G. (1996) Evaluation of a psychiatric court liaison scheme in North London. British Medical Journal, 313, 531-532.

ROSSAU, C. D. \& MORTENSEN, P. B. (1997) Risk factors for suicide in patients with schizophrenia: nested case-control study. British Journal of Psychiatry, 171, 355-359.

SNOWDEN, P., MCKENNA, J. \& JASPER, A. (1999) Management of conditionally discharged patients and others who present similar risks in the community: integrated or parallel? Journal of Forensic Psychiatry, 10, 583-596.

STEADMAN, H. J., MULVEY, E., MONAHAN, J., et al (1998) Violence by people discharged from acute psychiatric inpatient facilities and by others in the same neighborhoods. Archives of General Psychiatry, 55, 393-401.

SZMUKLER G (2000) Homicide inquiries. What sense do they make? Psychiatric Bulletin, 24, 6-10.

TAYLOR, P. J. \& GUNN, J. (1999) Homicides by people with menta illness: myth and reality. British Journal of Psychiatry, 274, 9-14. 